\title{
Direct visualization of swollen microgels by scanning electron microscopy using ionic liquids
}

\author{
Koji Horigome ${ }^{1}$, Takeshi Ueki ${ }^{2,4}$ and Daisuke Suzuki ${ }^{1,3}$
}

We developed a simple and versatile method for the visualization of swollen microgels using standard scanning electron microscopy (SEM) that does not require the conventional sample pretreatment steps of sputtering. Specifically, microgels were swollen using ionic liquids (ILs), which remained nonvolatile even under high vacuum conditions. Two types of widely studied stimuli-responsive microgels and their hybrids with Au nanoparticles were visualized via SEM to demonstrate the versatility of the method. In particular, we observed the dispersion of embedded Au nanoparticles within the microgels because of the swelling caused by the ILs, confirming that the approach is versatile and useful for the evaluation of nanocomposite materials, such as hybrid microgels.

Polymer Journal (2016) 48, 273-279; doi:10.1038/pj.2015.103; published online 28 October 2015

\section{INTRODUCTION}

Hydrogel particles (microgels), which are composed of crosslinked polymer networks that swell in aqueous solutions, range in size from $10 \mathrm{~nm}$ to several micrometers, and thus can be dispersed in solvents. ${ }^{1}$ In particular, an attractive feature of microgels is their responsiveness to external stimuli such as changes in $\mathrm{pH}$ or temperature., ${ }^{2,3}$ For instance, monodispersed, thermoresponsive microgels have been prepared using $N$-isopropylacrylamide (NIPAm), ${ }^{3,4}$ whereas $\mathrm{pH}$-responsive poly(4-vinylpyridine) microgels have been prepared by emulsion polymerization. ${ }^{5}$ By varying the temperature or $\mathrm{pH}$, these microgels exhibit reversible volumetric responses because of changes in the conformation of the constituent polymers within the microgels. These microgel properties can be employed in many applications, including drug delivery systems, ${ }^{6-8}$ chemical separations, ${ }^{9,10}$ smart particulate emulsifiers, ${ }^{11}$ photonic crystals ${ }^{12,13}$ and hybrid materials. ${ }^{14,15}$

To characterize microgels, visualization techniques such as optical and electron microscopy are frequently used. These methods are important tools for understanding the colloidal behavior and morphology of microgels because they enable the direct monitoring of these systems. ${ }^{2,16}$ Optical microscopy can be used to directly observe the Brownian motion and the structures of microgels in the swollen state. However, the effective resolution for optical analysis of microgels is $\sim 1 \mu \mathrm{m}$ because the wavelength of the light source is in the visible region, and highly swollen microgels have a refractive index that is close to that of water. ${ }^{17}$ To obtain morphological information about microgels at the submicron scale, electron microscopy techniques such as scanning electron microscopy (SEM) and transmission electron microscopy (TEM) are generally used. SEM is a common and useful method for the visualization of the surface morphology of submicron particles. ${ }^{18}$ Images of particles are obtained by scanning them with an electron beam and then detecting the secondary electrons scattered from the surfaces of the particles. However, SEM samples must be dried before analysis, and the sample chambers are maintained under high vacuum during the measurements. In addition, when nonconductive materials, such as microgels, are observed via SEM, a conductive layer, which can be formed by sputtering with $\mathrm{Au}$ or $\mathrm{Pt} / \mathrm{Pd}$ is required to suppress the accumulation of electrons that cause artifacts in the SEM images. Because microgels exhibit unique properties, such as stimuli responsiveness in the swollen state, an analysis method for the morphology of swollen submicron microgels using SEM is needed.

To overcome the limitations associated with the observation of swollen microgels via SEM, we focused on the use of ionic liquids (ILs), which exist in the liquid state at room temperature. Given the fascinating properties of ILs ${ }^{19-25}$ such as their low vapor pressure, ${ }^{21}$ high conductivity ${ }^{21}$ and high solubility for many monomers and polymers, ${ }^{22-24}$ it was thought that if microgels could be swollen using ILs under vacuum, it should be possible to observe the swollen microgels via SEM. Kuwabata et al. ${ }^{25}$ reported that many types of biological samples such as insects, flowers and cells can be observed via SEM by using IL coatings. However, it is still unclear whether the IL was introduced to the sample or adsorbed at the sample surface. The swollen state of the samples needs clarification. In addition, to the best of our knowledge, research has not been performed on the swelling behavior of submicron-sized polymer particles using ILs. Therefore, in this study, we examine the penetration of ILs into microgels and the direct visualization of submicron-sized microgels swollen using ILs

\footnotetext{
${ }^{1}$ Graduate School of Textile Science \& Technology, Shinshu University, Ueda, Japan; ${ }^{2}$ Department of Materials Engineering, School of Engineering, The University of Tokyo, Tokyo, Japan and ${ }^{3}$ Division of Smart Textiles, Institute for Fiber Engineering, Interdisciplinary Cluster for Cutting Edge Research, Shinshu University, Ueda, Japan Correspondence: Professor D Suzuki, Graduate School of Textile Science \& Technology, Shinshu University, 3-15-1, Tokida, Ueda, Nagano 386-8567, Japan. E-mail: d_suzuki@shinshu-u.ac.jp

${ }^{4}$ Current address: Polymer Materials Unit, National Institute for Materials Science, 1-1 Namiki, Tsukuba, Ibaraki 305-0044, Japan.

Received 7 July 2015; revised 1 September 2015; accepted 10 September 2015; published online 28 October 2015
} 
was investigated via SEM. To achieve this goal, two extensively explored stimuli-responsive microgels, poly(2-vinylpyridine) (denoted as p2VP) and poly( $N$-isopropylacrylamide) (denoted as pNIPAm), were selected. ${ }^{14,15,25}$

\section{EXPERIMENTAL PROCEDURE}

\section{Materials}

The reagents, including 2-vinylpyridine (2VP, 97\%), divinylbenzene (94-97\%, mixture of isomers), 2,2'-azobis(2-methylpropionamidine)dihydrochloride (V-50, 95\%), 2,2'-azobis(isobutyronitrile) (98\%), NIPAm (98\%), N, N'-methylenbis-(acrylamide) (BIS), glycidyl methacrylate (95\%), dimethylamine borane (97\%), 2-aminoethanethiol, hydroxylammonium chloride $\left(\mathrm{NH}_{2} \mathrm{OH} \cdot \mathrm{HCl}\right)$, hydrogen tetrachloroaurate(III) tetrahydrate $\left(\mathrm{HAuCl}_{4}\right), 0.1 \mathrm{~m}$ hydrochloric acid and methanol $(\mathrm{MeOH}, 99 \%)$, were purchased from Wako Pure Chemical Industries (Tokyo, Japan) and used as received. Water that was used in all reactions, solution preparation and polymer purification was first distilled and then ion-exchanged (EYELA, SA-2100E1, Tokyo Rikakikai (Tokyo, Japan)). A polystyrene dish (IWAKI, $60 \mathrm{~mm} /$ non-treated dish, Asahi Glass (Shizuoka, Japan)) was cut into $1 \times 1 \mathrm{~cm}^{2}$ or $0.5 \times 1 \mathrm{~cm}^{2}$ pieces using a cutter and then sputtered with $\mathrm{Pt} / \mathrm{Pd}$ under $6 \mathrm{~Pa}$ and $15 \mathrm{~mA}$ for $200 \mathrm{~s}$ before drying each sample. The distance between the sample and the sputtering target was adjusted to $30 \mathrm{~mm}$. The ILs, imidazole 1,1,1-trifluoro- $N$-[(trifluoromethyl) sulfonyl] methanesulfonamide $\left([\mathrm{Im}]\left[\mathrm{NTf}_{2}\right]\right)$ and diethylmethylamine trifluorosulfonate ([dema][TfO]), were prepared by neutralization of the appropriate Brønsted acid with the appropriate Brønsted base according to the literature procedure. ${ }^{26}$

\section{Preparation of p2VP microgels}

p2VP microgels were prepared by soap-free emulsion polymerization. First, water $(40 \mathrm{ml})$ was poured into a $50-\mathrm{ml}$ three-neck, round-bottom flask, and then $2 \mathrm{VP}(637 \mu \mathrm{l}, 150 \mathrm{~mm})$, divinylbenzene $(10 \mu \mathrm{l}, 150 \mathrm{~mm})$ and $\mathrm{NaCl}(0.023 \mathrm{~g}$, $10 \mathrm{~mm}$ ) were mixed into the water. The total monomer concentration was fixed at $150 \mathrm{~mm}$. Next, the flask that contained the monomer solution was equipped with a mechanical stirrer, a condenser and an inlet for nitrogen gas, and nitrogen gas was bubbled through the monomer solution for $30 \mathrm{~min}$ at $70^{\circ} \mathrm{C}$ to purge any oxygen. An aqueous solution of the V-50 initiator $(0.023 \mathrm{~g}$ in $5 \mathrm{ml})$ was then injected into the flask to begin the polymerization. Nitrogen gas was streamed through the flask for $1 \mathrm{~h}$, and the polymerization was continued for $24 \mathrm{~h}$ with constant stirring at 250 r.p.m. Next, the microgel dispersion was cooled to room temperature and purified using a centrifugation/redispersion process. An aliquot of the microgel dispersion was centrifuged with a relative centrifugal force of $1500 \mathrm{~g}$, (gravitational acceleration, $g$ : $9.8 \mathrm{~ms}^{-2}$ ); then, the supernatant was replaced with pure water. This purification process was repeated four times.

\section{Preparation of p2VP bulk gel}

The p2VP bulk gel was prepared by bulk polymerization. First, 2VP (521 $\mu$ l) and divinylbenzene $(8.4 \mu \mathrm{l})$ were added to a glass vial that contained $\mathrm{MeOH}$. Next, nitrogen gas was bubbled through the monomer solution for $15 \mathrm{~min}$; then, 2,2'-azobis(isobutyronitrile) $(0.013 \mathrm{~g})$ was dissolved in the solution. The mixture was injected in the space between two glass plates that were gapped with a silicone sheet. To initiate the polymerization, the solution was heated to $60^{\circ} \mathrm{C}$. The polymerization was maintained for $8.5 \mathrm{~h}$. To purify the bulk gel, the p2VP gel sheet was immersed in $\mathrm{MeOH}$. This purification process was repeated four times.

\section{Preparation of hybrid microgels}

A series of hybrid microgels were prepared using previously reported procedures. ${ }^{14,15}$ First, pNIPAm-co-glycidyl methacrylate microgels, which are templates for the synthesis of the Au nanoparticles, were obtained by soap-free emulsion copolymerization. Water $(590 \mathrm{~g})$ was placed into a $1000 \mathrm{ml}$ three-neck, round-bottom flask; then, NIPAm (11.25 g, $194 \mathrm{~mm})$, glycidyl methacrylate $(2.25 \mathrm{~g}, 194 \mathrm{~mm})$ and BIS $(0.18 \mathrm{~g}, 194 \mathrm{~mm})$ were added to the water. Next, the flask containing the monomer solution was equipped with a mechanical stirrer, a condenser and an inlet for nitrogen gas. Then, nitrogen gas was bubbled through the monomer for $30 \mathrm{~min}$ at $70^{\circ} \mathrm{C}$ to purge any oxygen.
An aqueous solution of the V-50 initiator $(0.36 \mathrm{~g}$ in $10 \mathrm{~g}$ of water) was injected into the flask to begin the polymerization. The polymerization was continued for $6 \mathrm{~h}$ with constant stirring at 250 r.p.m., after which the microgel dispersion was cooled to room temperature. The microgel dispersion was then purified using a centrifugation/redispersion process. An aliquot of the microgel dispersion was centrifuged with a relative centrifugal force of $50000 \mathrm{~g}$. Then, the supernatant was replaced with pure water. This purification process was repeated four times.

Next, the epoxy groups in the glycidyl methacrylate units within the microgels were reacted with the thiol groups of 2-aminoethanethiol to introduce amino groups into the microgels. ${ }^{15}$ Water was added to the 2-aminoethanethiol $(1.6 \mathrm{~g})$ in a glass vial. Then, the $\mathrm{pH}$ of the solution was adjusted to $>11$ by adding $1 \mathrm{~m} \mathrm{NaOH}(3 \mathrm{ml})$. The microgel dispersion (1.0 wt.\%) was then mixed into this solution, and the amination reaction was continued for $24 \mathrm{~h}$. The obtained amino-functionalized microgels were purified by centrifugation/redispersion with water six times, followed by dialysis for 3 days. After purification of the amino-functionalized microgels, the in situ synthesis of the Au nanoparticles within the microgels was achieved through the reduction of $\mathrm{HAuCl}_{4}$ that was adsorbed onto the microgels by electrostatic attraction to the amino groups. A mixture of amino-functionalized microgels $(50 \mathrm{mg})$ and $\mathrm{HAuCl}_{4}(4 \mathrm{mg})$ was stirred in an aqueous medium $(\mathrm{pH} 3,10 \mathrm{ml})$ at $4{ }^{\circ} \mathrm{C}$ for $4 \mathrm{~h}$. Subsequently, the excess $\mathrm{HAuCl}_{4}$ was removed by centrifugation/redispersion with water twice. The $\mathrm{pH} 3$ aqueous solution $(20 \mathrm{ml}$ total) containing the microgels was placed in a $30-\mathrm{ml}$ glass vial at $4{ }^{\circ} \mathrm{C}$. Separately, $\mathrm{NaBH}_{4}(20 \mathrm{mg})$ was dissolved in water $(20 \mathrm{ml})$. This solution was then added dropwise to the vial containing the microgel, and the mixture was stirred for 30 min. The pNIPAm-based microgels with the Au nanoparticles were then purified by centrifugation/redispersion with water four times.

In addition, the $\mathrm{Au}$ nanoparticles embedded within the microgels were grown using an electroless plating process to easily characterize them via field emission SEM (FE-SEM). A mixture of the microgel $(2.5 \mathrm{mg})$ and water $(20 \mathrm{ml}$ total) was placed in a $30-\mathrm{ml}$ glass vial and stirred at $4{ }^{\circ} \mathrm{C}$. After $10 \mathrm{~min}$ of stirring, a mixture of $\mathrm{HAuCl}_{4}(1 \mathrm{wt} . \%, 200 \mu \mathrm{l})$ and $\mathrm{NH}_{2} \mathrm{OH} \cdot \mathrm{HCl}(40 \mathrm{~mm}$, $1000 \mu \mathrm{l})$ was poured into the vial. The reaction continued for $24 \mathrm{~h}$, after which the microgels were purified by centrifugation/redispersion twice with water.

\section{Characterization of microgel size}

The hydrodynamic diameter of the microgels was characterized using dynamic light scattering (DLS; Malvern Instruments (Tokyo, Japan), Zetasizer Nano S). An average of 15 measurements with 30-s acquisition times was used for the DLS data. The microgel concentration was fixed at $\sim 0.005$ wt. $\%, 0.1 \mathrm{M} \mathrm{NaCl}$ was used to adjust the salt concentration to $1 \mathrm{~mm}$, and $5 \mathrm{M} \mathrm{NaCl}$ was used to adjust the salt concentration to $1 \mathrm{~m}$. When the hydrodynamic diameter was measured under acidic conditions, $10 \mu \mathrm{HCl}(0.1 \mathrm{M})$ was added to the solution to adjust the $\mathrm{pH}$ to $\sim 3$. The samples were allowed to thermally equilibrate at $25^{\circ} \mathrm{C}$ for $10 \mathrm{~min}$ before measurement, and the scattered light was collected at $173^{\circ}$. The hydrodynamic diameter of the microgels was determined from the measured diffusion coefficients using the Stokes-Einstein equation (Zetasizer software v6.12, Malvern Instruments). The $\mathrm{pH}$ was measured using a D-54SE (Horiba, Kyoto, Japan) instrument equipped with a $\mathrm{pH}$ electrode (Horiba 9618-10D) after each DLS measurement. The sizes of the microgels were also characterized based on analysis of the SEM or FE-SEM images using ImageJ (ver. 1.47t) software (Rasband WS. National Institutes of Health, Bethesda, MD, USA). The average size was determined from 30 measurements. The coefficient of variation $(\mathrm{CV})$ of the microgels was calculated using the following equation: $\mathrm{CV}=\sigma / A$, where $\sigma$ is the root-mean-square deviation of the microgel size, which was calculated for 30 measurements, and $A$ is the arithmetic mean of the microgel size $(N=30)$.

\section{SEM/FE-SEM observation of the microgels}

First, to suppress the accumulation of electrons, the polystyrene substrate was sputtered with $\mathrm{Pt} / \mathrm{Pd}$ for $200 \mathrm{~s}$ at $6 \mathrm{~Pa}$ and $15 \mathrm{~mA}$ before drying the dispersion. The distance between the sample and the sputtering target was fixed at $30 \mathrm{~mm}$. Under these conditions, a $\sim 20 \mathrm{~nm} \mathrm{Pt/Pd}$ layer was fabricated on the polystyrene substrate. The microgel dispersion (concentration: 0.001 wt.\%) was then deposited on the $\mathrm{Pt} / \mathrm{Pd}$ substrate and dried at room temperature 
$\left(25 \pm 2{ }^{\circ} \mathrm{C}\right)$. After drying, the sample was sputtered with $\mathrm{Pt} / \mathrm{Pd}$ for $80 \mathrm{~s}$ at $6 \mathrm{~Pa}$ and $15 \mathrm{~mA}$. The distance between the sample and the sputtering target was fixed at $30 \mathrm{~mm}$. Under these conditions, an $\sim 8 \mathrm{~nm} \mathrm{Pt} / \mathrm{Pd}$ layer was fabricated on the polystyrene substrate. The samples were observed via SEM (Hitachi, S-3000N (Tokyo, Japan)) and FE-SEM (Hitachi, S-5000 (Tokyo, Japan)) at a $20 \mathrm{keV}$ accelerating voltage.

\section{SEM/FE-SEM observation of the microgels using ionic liquids}

The common water-soluble ILs $[\mathrm{Im}]\left[\mathrm{NTf}_{2}\right]$ and [dema][TfO] were used for the SEM and FE-SEM observations. First, the IL was dissolved in water in a $1.5 \mathrm{ml}$ tube, and then the microgel dispersion $(1 \mathrm{ml}, 0.001 \mathrm{wt} . \%)$ was dispersed into the solution. A mixer (Eppendorf (Hamburg, Germany), Thermomixer comfort) was used to homogenize the mixture at 1000 r.p.m. and $\sim 25^{\circ} \mathrm{C}$ for $10 \mathrm{~min}$. The mixture was then deposited on the $\mathrm{Pt} / \mathrm{Pd}$ substrate, and the dispersion was dried at room temperature $\left(25 \pm 1.5^{\circ} \mathrm{C}\right)$. The SEM and FE-SEM measurements without $\mathrm{Pt} / \mathrm{Pd}$ sputtering of the sample were then immediately performed at an accelerating voltage of $20 \mathrm{keV}$. The images were analyzed using Image (ver. 1.47t) software.

\section{Procedure for profiling the samples}

First, the greyscale values of the FE-SEM images were fixed between 0 and 255 to select an 8-bit image, and then the microgels were profiled with the Image) software using the contrast along a line with a length of $0.7 \mu \mathrm{m}$. The lines were adjusted to cover the entire microgel. The average contrast for the $\mathrm{Pt} / \mathrm{Pd}$ substrate was also estimated by profiling the substrate along the $0.7 \mu \mathrm{m}$ line, and then the average contrasts were calculated using the greyscale values.

\section{RESULTS AND DISCUSSION}

First, pH-responsive p2VP microgels were prepared by soap-free emulsion polymerization, as previously reported. ${ }^{27}$ The size and $\mathrm{pH}$-responsive properties of the $\mathrm{p} 2 \mathrm{VP}$ microgels were evaluated using DLS. Because the pKa of the p2VP microgels is $\sim 4.1,{ }^{27}$ they swell at pH 3.3 and de-swell at $\mathrm{pH}$ 6.4. When the total salt concentration was fixed at $1 \mathrm{~mm}$, the hydrodynamic diameter $D_{\mathrm{h}}$ was $1347 \mathrm{~nm}$ at $\mathrm{pH}$ $3.3\left(D_{\mathrm{h}-\mathrm{pH} 3}\right)$ and $234 \mathrm{~nm}$ at $\mathrm{pH} 6.4\left(D_{\mathrm{h}-\mathrm{pH} 6}\right)$. The polydispersity index of the p2VP microgels was 0.152 and 0.024 , respectively. Next, to confirm the monodispersity, the dried p2VP microgels were observed via $\mathrm{FE}-\mathrm{SEM}$ with $\mathrm{Pt} / \mathrm{Pd}$ sputtering, which is the standard method for electron microscopic observation (Figure 1a). When the microgels were observed via SEM, the p2VP microgels could also be observed (Supplementary Figure 1). Because the FE-SEM images were clearer than the SEM images (Figure 1 vs Supplementary Figure 1, respectively), the FE-SEM images were used for the characterization. In Figure 1a, the p2VP microgels were deformed on the substrate, and they showed a hemispherical morphology. The deformation of the p2VP microgels was due to the evaporation of water from the microgels and adsorption on the $\mathrm{Pt} / \mathrm{Pd}$ substrate. The average size of the dried p2VP microgels $\left(D_{\text {dry }}\right)$ was $245 \pm 13 \mathrm{~nm}(N=30)$, and the $\mathrm{CV}$ was $5.3 \%$. Therefore, it was concluded that monodispersed p2VP microgels were obtained.

The effect of the ILs on the p2VP microgels was then investigated. The IL imidazole $[\mathrm{Im}]\left[\mathrm{NTf}_{2}\right]$, which is well-known as a protic watersoluble IL, was used for the FE-SEM measurements because p2VP microgels are swollen under acidic conditions. For sample preparation, a mixture of a p2VP microgel dispersion and $[\mathrm{Im}]\left[\mathrm{NTf}_{2}\right]$ (ca. $10 \mu \mathrm{l}$ total) was dried using the procedure shown in Figure 2. First, [Im] $\left[\mathrm{NTf}_{2}\right]$ was dissolved in water; then, the $\mathrm{p} 2 \mathrm{VP}$ microgel dispersion was added to the mixture to achieve a concentration of $0.001 \mathrm{wt} . \%$. Next, the dispersion was deposited onto the conductive substrate (that is, a polystyrene substrate sputtered with $\mathrm{Pt} / \mathrm{Pd}$ ) and dried at room temperature $\left(25 \pm 1.5^{\circ} \mathrm{C}\right)$. After the complete removal of water, the sample was directly observed via FE-SEM without sputtering onto the microgels. To determine the amount of IL required for the observation, samples with varying amounts of added $[\mathrm{Im}]\left[\mathrm{NTf}_{2}\right](0.5,1,2$ and $5 \mu \mathrm{l}$ ) were investigated. In all cases, it was found that pools of the IL appeared (Supplementary Figure 2b), and the p2VP microgels were embedded in the pools. The size of the pools decreased as the amount of added $[\mathrm{Im}]\left[\mathrm{NTf}_{2}\right]$ decreased, and when $0.5 \mu \mathrm{l}$ of $[\mathrm{Im}]\left[\mathrm{NTf}_{2}\right]$ was dissolved into $1 \mathrm{ml}$ of water, the p2VP microgels were observed near the pools, as shown in Figure 1b. We believe that this FE-SEM image represents the first observation of a submicron-sized, polymeric microgel that is swollen using an IL without sputtering. The swelling state of IL-swollen p2VP microgels is discussed in the next paragraph. Charging did not occur during the observation. As a control experiment, charging was observed on the microgels when the IL was not added to the dispersion before drying (Supplementary Figure 3). These results suggest that primary electrons irradiated from the electron gun are not accumulated on the IL-swollen microgels. Therefore, the IL behaves like an electronically conducting material. ${ }^{25}$ To the best of our knowledge, the effect of the SEM visualization mechanism in ionic liquids is still unclear and is under investigation by several researchers. Wishart and $\mathrm{Neta}^{28}$ have confirmed that electrons are remarkably stable under a high ion concentration atmosphere, such as in an ionic liquid, in terms of systematic pulse radiolysis measurements for a series of ionic liquids. It is generally speculated that electrons stabilized in an ionic liquid assist in generating secondary electrons from ionic liquid-coated samples, which results in high-resolution SEM images without charging. ${ }^{29}$ Kuwabata's group has further reported an important control experiment in which a nonvolatile silicon oil without any conductivity exhibited a white image with much noise. By contrast, an ionic liquid droplet produced an SEM image without any white noise.
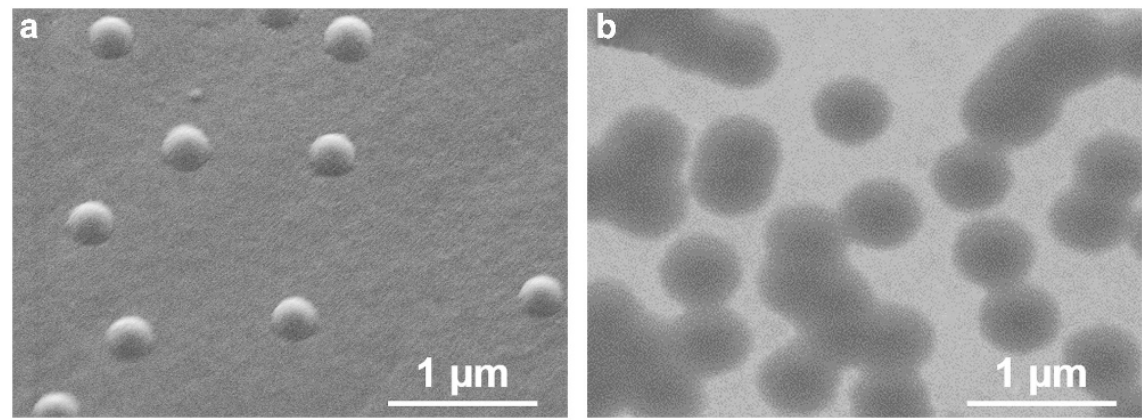

Figure 1 Field-emission scanning electron microscopy images of (a) dried microgels and (b) ionic liquid-swollen microgels. In both cases, $10 \mu \mathrm{l}$ of 0.001 wt. $\%$ microgels were dried at $24.5^{\circ} \mathrm{C}$ on a Pt/Pd substrate, and the sample stage was tilted at $40^{\circ}$. (b) The microgels were swollen using $0.5 \mu \mathrm{l}$ of [Im][NTf $\left.{ }_{2}\right]$ (imidazole 1,1,1-trifluoro- $N$-[(trifluoromethyl) sulfonyl] methanesulfonamide). 


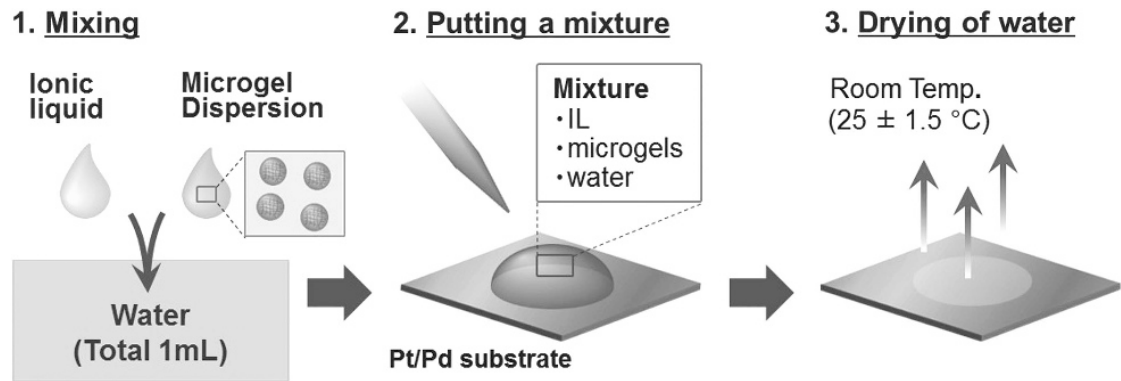

Figure 2 Schematic of the sample preparation for scanning electron microscopy or field emission SEM measurements of the ionic liquid (IL)-swollen microgels. A full color version of this figure is available at Polymer Journal online.
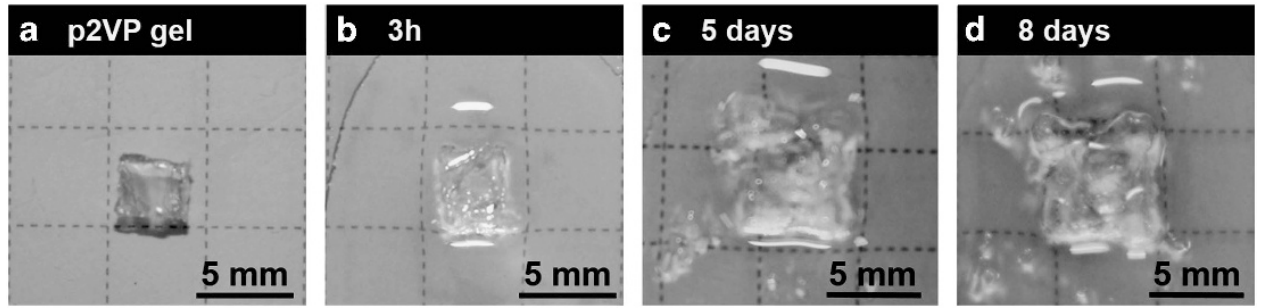

Figure 3 (a) A photograph of the poly(2-vinylpyridine) (p2VP) bulk gel. [Im][NTf ${ }_{2}$ (imidazole 1,1,1-trifluoro- $N$-[(trifluoromethyl) sulfonyl] methanesulfonamide) was dropped onto the p2VP bulk gel, and the bulk gel was observed at (b) $3 \mathrm{~h}$, (c) 5 days and (d) 8 days. A full color version of this figure is available at Polymer Journal online.

This result implies that the high ion concentration atmosphere of an ionic liquid is not charged and has a crucial role in SEM visualization. ${ }^{25}$ In addition, metal nanoparticles containing an ionic liquid are easily observable by TEM. This also suggests that the electron beam can easily penetrate the ionic liquid. ${ }^{30}$ Therefore, in our system, the electrons that are irradiated by the electron gun may penetrate $[\operatorname{Im}][\mathrm{NTf} 2]$ and [dema][TfO], as described above. Note that only a small quantity of $[\mathrm{Im}]\left[\mathrm{NTf}_{2}\right](\sim 0.5 \mu \mathrm{l})$ was required to achieve the FE-SEM observation of the nonelectrically conducting microgels.

To determine the swelling ratio of the IL-swollen p2VP microgels, the size of the particles was estimated from the FE-SEM image (Figure 1b). The average diameter of the IL-swollen p2VP microgels $\left(D_{\mathrm{IL}}\right)$ was $523 \pm 39 \mathrm{~nm}(N=30)$, and the $\mathrm{CV}$ was $7.4 \%$. Thus, the $D_{\text {IL }}$ was $\sim 2.0$ times as large as that of the dried p2VP microgels ( $D_{\text {dry: }}: 245 \mathrm{~nm}$ ), but it was only $\sim 0.4$ times the size of the highly swollen p2VP microgels in an acidic solution $\left(D_{\mathrm{h}-\mathrm{pH}}: 1347 \mathrm{~nm}\right)$. Therefore, the p2VP microgels were swollen with $[\operatorname{Im}]\left[\mathrm{NTf}_{2}\right]$ but not to the fully swollen state $\left(D_{\mathrm{h}-\mathrm{pH} 3}\right)$. This insufficient swelling of the p2VP microgels by $[\mathrm{Im}]\left[\mathrm{NTf}_{2}\right]$ may be due to the high ionic strength of the IL. Thus, to evaluate the effect of the IL ionic strength on the $D_{\mathrm{h}}$, DLS analysis was performed for the p2VP microgels at a high salt concentration (that is, $1 \mathrm{M} \mathrm{NaCl}$ ) at $\mathrm{pH} 3.1\left(D_{\mathrm{h}-\mathrm{pH}-1 \mathrm{MNaCl}}\right)$. Under these conditions, the $D_{\mathrm{h}}$ was $677 \mathrm{~nm}$, which is close to the $D_{\mathrm{IL}}$. The size data for the evaluated p2VP microgels are summarized in Supplementary Table 1. On the basis of these results, the insufficient swelling can be explained by the charge screening effect of [Im] $\left[\mathrm{NTf}_{2}\right]$, which is similar to the effect of $\mathrm{NaCl}$.

In addition, to characterize the swelling of the p2VP networks, [Im] $\left[\mathrm{NTf}_{2}\right]$ was deposited onto a dried p2VP bulk gel, and the swelling behavior of the p2VP bulk gel was observed with the naked eye (Figure 3). The preparation of the p2VP bulk gels is described in the Experimental Procedure section. When the p2VP bulk gel was observed after 8 days, the size of the bulk gel was bigger than that of the dried bulk gel (Figure $3 \mathrm{a}$ vs d, respectively). The p2VP bulk gel was clearly swollen with $[\mathrm{Im}]\left[\mathrm{NTf}_{2}\right]$. Then, to determine the water content inside the microgels, it was calculated by measuring the weight before and after drying the mixture of the microgel dispersion with $[\mathrm{Im}]\left[\mathrm{NTf}_{2}\right]$. The result is summarized in Supplementary Table 2. The water content of the p2VP microgel after drying was calculated to be $0.1 \%$, indicating that the microgel was filled with the IL. Thus, it is clear that the p2VP microgels were indeed swollen by the IL during SEM observation.

Next, to confirm the versatility of the method, microgels that were mainly composed of pNIPAm were observed using diethylmethylamine trifluorosulfonate ([dema][TfO]), which is another common, protic and water-soluble ionic liquid. ${ }^{31}$ Notably, when $[\mathrm{Im}]\left[\mathrm{NTf}_{2}\right]$ was used for the observation, the pNIPAm microgels were not swollen (Supplementary Figure 4; this may be due to the solubility related to the anion of the ILs). ${ }^{32}$ Thus, to obtain good affinity between the microgels and the IL, the chemical composition of both systems must be carefully considered. In this case, to characterize the swelling of the pNIPAm microgels in more detail, Au nanoparticles were synthesized within the pNIPAm microgels as indicators of the expansion of the microgels. Thus, if the pNIPAm microgels containing the $\mathrm{Au}$ nanoparticles (hybrid microgel) were swollen by [dema][TfO], the $\mathrm{Au}$ nanoparticles that were embedded within the microgels would be individually dispersed throughout the microgels. The hybrid microgels were prepared according to the procedure reported by Suzuki and Kawaguchi. ${ }^{14,15}$ To determine the size and monodispersity of the hybrid microgels, a microgel dispersion was dried at room temperature, and then the sample was sputtered with $\mathrm{Pt} / \mathrm{Pd}$ and observed via FE-SEM (Figure 4a). In this case, FE-SEM was necessary for clear observation of the Au nanoparticles. As shown in Figure 4a, the Au nanoparticles (bright dots) were localized at the center of the microgels. Notably, the size of the dried hybrid microgels was $372 \pm 25 \mathrm{~nm}(N=30)$, and the $\mathrm{CV}$ was $6.7 \%$. By contrast, when a mixture of the hybrid microgel dispersion $(9 \mu \mathrm{l})$ and [dema][TfO] $(1 \mu \mathrm{l})$ was dried on a conductive substrate and observed via FE-SEM without $\mathrm{Pt} / \mathrm{Pd}$ sputtering (Figure $4 \mathrm{~b}$ ), the size of the hybrid microgels increased to $555 \pm 43 \mathrm{~nm}(N=30)$, and the $\mathrm{CV}$ was $7.7 \%$. Then, $\mathrm{Au}$ 

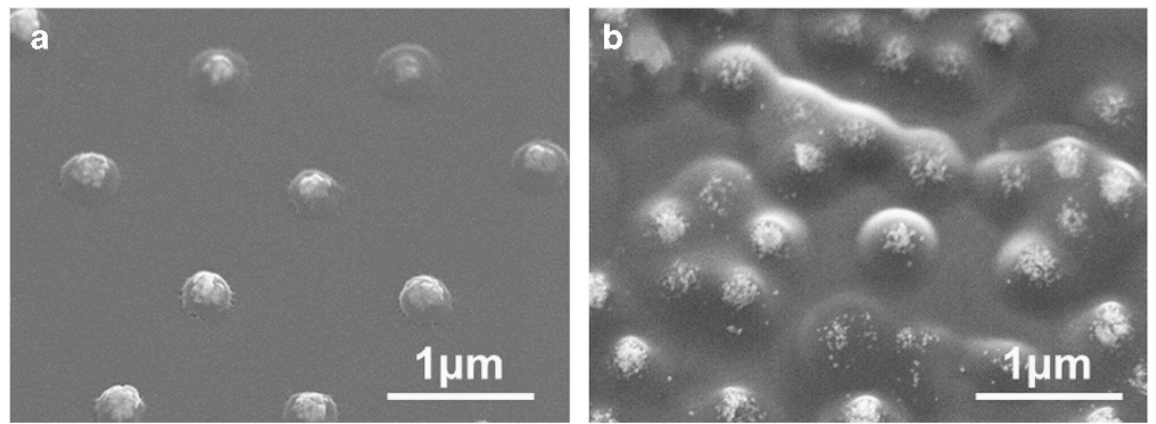

Figure 4 Field-emission scanning electron microscopy images of the hybrid microgels containing the Au nanoparticles. (a) Pt/Pd sputtering was performed before the observation. (b) The microgels were swollen using $0.5 \mu$ l of [dema][T $\mathrm{O} O$ ] (diethylmethylamine trifluorosulfonate). In both cases, $10 \mu \mathrm{l}$ of a $0.001 \mathrm{wt}$. $\%$ microgel dispersion was dried at $24^{\circ} \mathrm{C}$ on a $\mathrm{Pt} / \mathrm{Pd}$ substrate, and the sample stage was tilted at $40^{\circ}$.
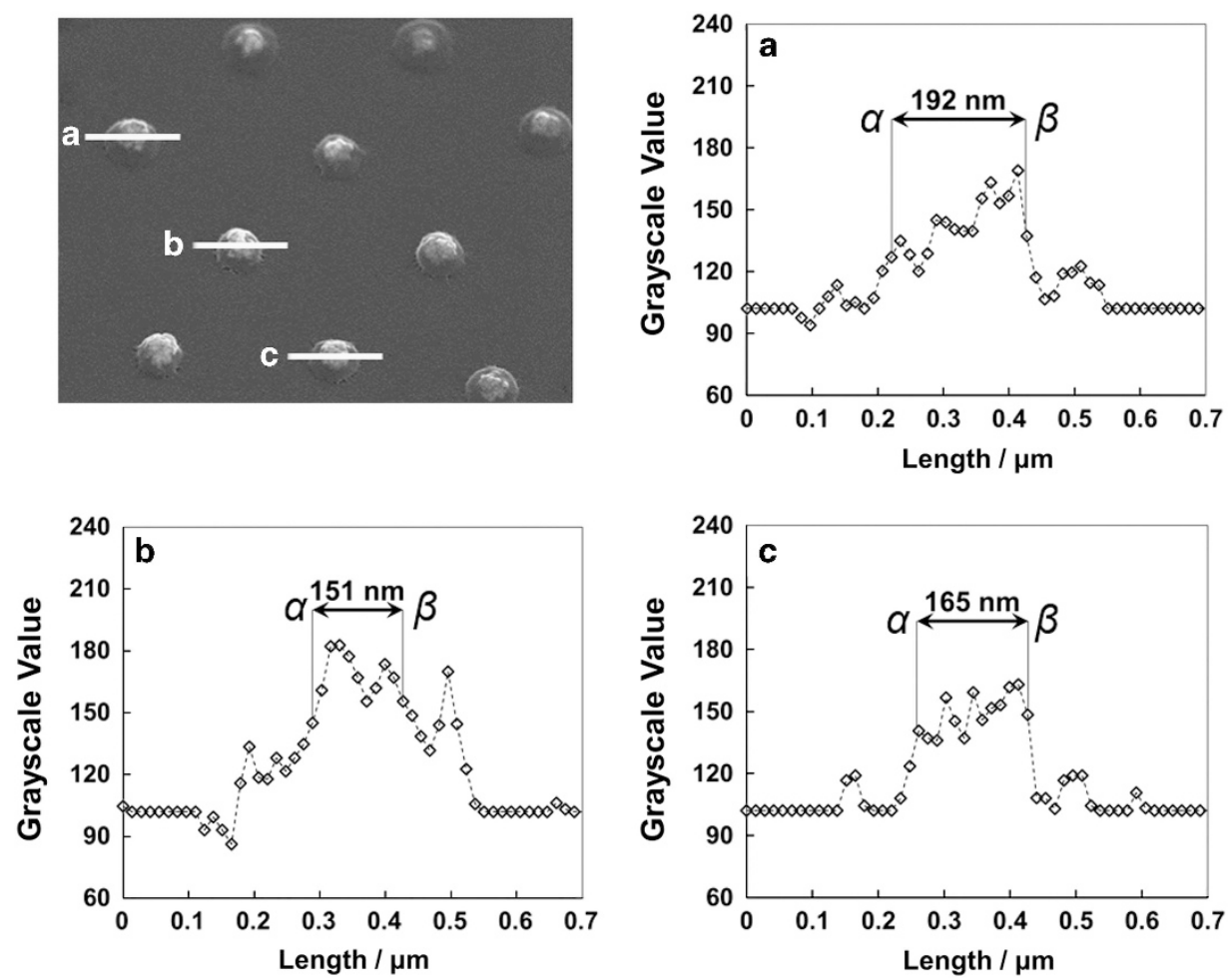

Figure 5 Field-emission scanning electron microscopy (FE-SEM) image and averaged greyscale value data for three dried hybrid pNIPAm microgels. The greyscale values were fixed between 0 and 255 using ImageJ software. (a), (b) and (c) show the greyscale values along the white solid lines (a), (b) and (c), respectively, as indicated in the FE-SEM image. The widths of the located Au nanoparticles (between $\alpha$ and $\beta$ ) were estimated to be (a) 192 , (b) 151 and (c) $165 \mathrm{~nm}$. The average width was $169 \mathrm{~nm}$.

nanoparticles were observed within the microgels, and the size was calculated to be $29 \mathrm{~nm}(N=30)$, which is similar to that calculated from a TEM image $(26 \mathrm{~nm}, N=30$; Supplementary Figure 5). Although SEM is generally used for characterization of surface morphology, Au nanoparticles located within the microgels could be observed. The water content of the pNIPAm microgels after drying was calculated to be $0.27 \%$ (Supplementary Table 3 ). Then, the pNIPAm microgels were swollen with [dema][TfO]. To characterize the swollen state of the microgels in more detail, the topography of dried and IL-swollen microgels was obtained using atomic force microscopy (Supplementary Figures 6 and 7). The experimental details are described in the supporting information. The measurements were in good agreement with the FE-SEM data. The cross-sections showed hemispherical images. The size of the IL-swollen microgels was larger than that of the dried microgels, and the diameter of the IL-swollen microgels was larger than the height of the IL-swollen microgels. This indicates that the microgels were swollen with the IL, and the IL-swollen microgels deformed on the substrate. The atomic force microscopy measurements supported the swelling of the microgels. The swelling ratio $(\varphi)$ of the pNIPAm microgels was then roughly estimated using the following equation:

$$
\varphi=V_{\mathrm{IL}-\mathrm{swollen}} / V_{\mathrm{dry}},
$$

where $V_{\text {IL-swollen }}$ is the volume of the IL-swollen hybrid microgels that are shown in Figure $4 \mathrm{~b}$. $V_{0}$ is the volume of the dried hybrid microgels that are shown in Figure $4 \mathrm{a}$. $V_{\text {IL-swollen }}$ and $V_{0}$ were estimated using atomic force microscopy (Supplementary Figure 6). Using the equation, the $\varphi$ of the hybrid microgels swollen by [dema][TfO] 

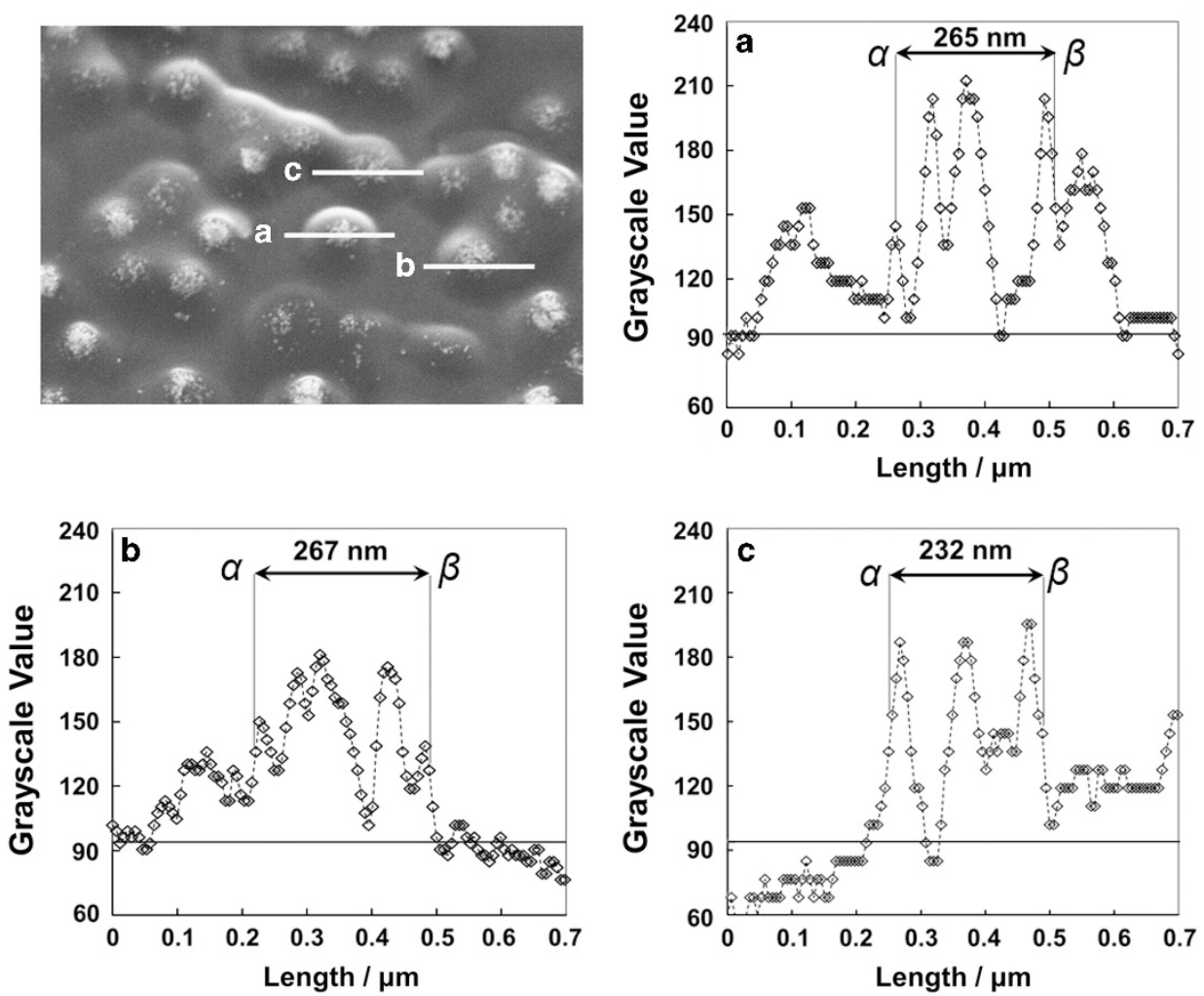

Figure 6 Field-emission scanning electron microscopy (FE-SEM) image and averaged greyscale value data for three ionic liquid (IL)-swollen hybrid pNIPAm microgels. The greyscale values were fixed between 0 and 255 using ImageJ software. The black solid lines, as shown in each greyscale value graph ((a), (b) and (c)), indicate the average brightness, which was estimated along the substrate using ImageJ software. Graphs (a), (b) and (c) show the greyscale values along the white solid lines (a), (b) and (c), respectively, as indicated in the FE-SEM image. The distances at which the Au nanoparticles were located (between $\alpha$ and $\beta$ ) were estimated to be (a) 265, (b) 267 and (c) $232 \mathrm{~nm}$ by calculating the lateral axis. The average distance was $254 \mathrm{~nm}$.

was determined to be 3.82. This indicates the swelling of the microgels, and the method shown in Figure 2 can also be applied to hybrid materials that are composed of pNIPAm microgels and Au nanoparticles.

Note that the bright dots were observed at the center of the microgels in Figure 4, indicating the presence of $\mathrm{Au}$ nanoparticles because $\mathrm{Au}$ nanoparticles have a much higher atomic weight compared with those of carbon, nitrogen and oxygen, which are the constituent elements of the pNIPAm microgel and [dema][TfO]. To characterize the dispersion of these Au nanoparticles within the microgels, the FE-SEM images shown in Figure 4 were converted to greyscale values (Figures 5 and 6). Details of the characterization method are described in the Experimental Procedure section. The bright region between $\alpha$ and $\beta$, as shown in Figures 5 and 6, indicates the presence of $\mathrm{Au}$ nanoparticles. Compared with the dried hybrid microgel (Figure 4a), the IL-swollen hybrid microgel (Figure 4b) was larger and the region in which the Au nanoparticles were present was wider (Figures 5 vs 6 , respectively). The average widths of the bright region for three measurements were 169 and $254 \mathrm{~nm}$ (Figures 5 and 6, respectively). Therefore, the area containing the Au nanoparticles in the IL-swollen hybrid microgel was $\sim 85 \mathrm{~nm}$ broader than that of the dried hybrid microgel. Thus, we conclude that the hybrid microgel was swollen by the [dema][TfO], causing the embedded Au nanoparticles to be dispersed within the microgel.

\section{CONCLUSION}

In conclusion, for the first time, we performed SEM and FE-SEM observation of the widely studied, stimuli-responsive, submicron-sized
p2VP and hybrid pNIPAm microgels using water-soluble ILs. Using this method, the sputtering of the samples and the associated special instrumentation such as cryogenic-SEM, ${ }^{33}$ cryogenic-TEM ${ }^{34}$ and scanning transmission $\mathrm{X}$-ray microscopy ${ }^{35,36}$ are not required. In addition, only a small amount of the IL (ca. $0.5 \mu \mathrm{l}$ ) is needed during sample preparation to swell the microgels. Au nanoparticles, which are located within the microgels, were observed via FE-SEM using [dema] [TfO], and the area containing the Au nanoparticles in the IL-swollen hybrid microgel was $\sim 85 \mathrm{~nm}$ broader than that of the dried hybrid microgel. Therefore, the microgels are indeed swollen by containing a good solvent, and this visualization method is useful to evaluate the internal structure of metal composite gel materials. In addition, chemical reactions can be applied within IL-swollen microgels. ${ }^{30}$ Therefore, the method is useful for in situ synthesis of hybrid gel materials. We believe that this simple method will become a standard technique for SEM observation of submicron-sized gel particles and will lead to new findings and a greater understanding of the structures of nanocomposites, such as hybrid microgels.

\section{CONFLICT OF INTEREST}

The authors declare no conflict of interest.

\section{ACKNOWLEDGEMENTS}

DS acknowledges Grant-in-Aids for (1) Challenging Exploratory Research (26620177) and (2) Scientific Research on Innovative Areas (26102517) from the Ministry of Education, Culture, Sports, Science and Technology of Japan. $\mathrm{KH}$ acknowledges the Research Fellowships of the Japan Society for the Promotion of Science for young Scientists. Moreover, the authors thank 
Yasuhisa Nagase for his help in preparing the hybrid microgels and TEM observations.

1 Lyon, L. A. \& Fernandez-Nieves, A. The polymer/colloid duality of microgel suspensions. Annu. Rev. Phys. Chem. 63, 25-43 (2012).

2 Saunders, B. R. \& Vincent, B. Microgel particles as model colloids: theory, properties and applications. Adv. Colloid Interface Sci. 80, 1-25 (1999).

3 Pelton, R. H. \& Chibante, P. Preparation of aqueous latices with $\mathrm{N}$-Isopropylacrylamide. Colloids Surf. 20, 247-256 (1986).

4 Pelton, R. Temperature-sensitive aqueous microgels. Adv. Colloid Interface Sci. 85 1-33 (2000).

$5 \mathrm{Ma}$, G. H. \& Fukutomi, T. Studies on the preparation and characterization of poly(4-vinylpyridine) microgel. I. preparation with polymer emulsifier. J. Appl. Polym. Sci. 43, 1451-1457 (1991).

6 Hsiue, G. H., Hsu, S. H., Yang, C. C., Lee, S. H. \& Yang, I. K. Preparation of controlled release ophthalmic drops, for glaucoma therapy using thermosensitive poly- $\mathrm{N}$-isopropylacrylamide. Biomaterials 23, 457-462 (2002).

7 Nayak, S., Lee, H., Chmielewski, J. \& Lyon, L. A. Folate-mediated cell targeting and cytotoxicity using thermoresponsive microgels. J. Am. Chem. Soc. 126, 10258-10259 (2004).

8 Smeets, N. M. B. \& Hoare, T. Designing responsive microgels for drug delivery applications. J. Polym. Sci. A: Polym. Chem. 51, 3027-3043 (2013).

9 Kawaguchi, H., Fujimoto, K. \& Mizuhara, Y. Hydrogel microspheres III. temperaturedependent adsorption of proteins on poly- $\mathrm{N}$-isopropylacrylamide hydrogel microspheres. Colloid Polym. Sci. 270, 53-57 (1992).

10 Parasuraman, D., Sarker, A. K. \& Serpe, M. J. Recyclability of poly ( $\mathrm{N}$-isopropylacrylamide) microgel-based assemblies for organic dye removal from water. Colloid Polym. Sci. 291, 1795-1802 (2013).

11 Wiese, S., Spiess, A. C. \& Richtering, W. Microgel-stabilized smart emulsions for biocatalysis. Angew. Chem. Int. Ed. 52, 576-579 (2013).

12 Reese, C. E., Mikhonin, A. V., Kamenjicki, M., Tikhonov, A. \& Asher, S. A. Nanoge nanosecond photonic crystal optical switching. J. Am. Chem. Soc. 126, 1493-1496 (2004).

13 Weissman, J. M., Sunkara, H. B., Tse, A. S. \& Asher, S. A. Thermally switchable periodicities and diffraction from mesoscopically ordered materials. Science 274, 959-960 (1996).

14 Suzuki, D. \& Kawaguchi, H. Stimuli-sensitive core/shell template particles for immobilizing inorganic nanoparticles in the core. Colloid Polym. Sci. 284, 1443-1451 (2006).

15 Suzuki, D. \& Kawaguchi, H. Hybrid microgels with reversibly changeable multiple brilliant color. Langmuir 22, 3818-3822 (2006).

16 Cohin, Y, Fisson, M, Jourde, K, Fuller, G. G, Sanson, N, Talini, L \& Monteux, C. Tracking the interfacial dynamics of PNiPAM soft microgels particles adsorbed at the air-water interface and in thin liquid films. Rheol. Acta 52, 445-454 (2013).

17 Jeenanong, A. \& Kawaguchi, H. Effect of $\mathrm{pH}$ and temperature on the behavior of microgel in SPR sensor. Colloids Surf. A 315, 232-240 (2008).
18 Crowther, H. M. \& Vincent, B. Swelling behavior of poly- $N$-isopropylacrylamide microgel particles in alcoholic solutions. Colloid Polym. Sci. 276, 46-51 (1998).

19 Ueki, T. \& Watanabe, M. Macromolecules in ionic liquids: progress, challenges, and opportunities. Macromolecules 41, 3739-3749 (2008).

20 Torimoto, T., Tsuda, T., Okazaki, K. \& Kuwabata, S. New frontiers in materials science opened by ionic liquids. Adv. Mater. 21, 1-26 (2009).

21 Plechkova, N. V. \& Seddon, K. R. Applications of ionic liquids in the chemical industry. Chem. Soc. Rev. 37, 123-150 (2008).

22 Minami, H., Yoshida, K. \& Okubo, M. Preparation of polystyrene particles by dispersion polymerization in an ionic liquid. Macromol. Rapid Commun. 29, 567-572 (2008).

23 Minami, H., Kimura, A., Kinoshita, K. \& Okubo, M. Preparation of poly(acrylic acid) particles by dispersion polymerization in an ionic liquid. Langmuir 26, 6303-6307 (2010).

24 Ueki, T. \& Watanabe, M. Polymers in ionic liquids: Dawn of neoteric solvents and innovative materials. Bull. Chem. Soc. Jpn. 85, 33-50 (2012).

25 Kuwabata, S., Kongkanand, A., Oyamatsu, D. \& Torimoto, T. Observation of ionic liquid by scanning electron microscope. Chem. Lett. 35, 600-601 (2006).

26 Noda, A., Susan, M. A. B. H., Kudo, K., Mitsushima, S., Hayamizu, K. \& Watanabe, M Brønsted acid-base ionic liquids as proton-conducting nonaqueous electrolytes. J. Phys. Chem. B 107, 4024-4033 (2003).

27 Dupin, D., Fujii, S. \& Armes, S. P. Efficient synthesis of sterically stabilized $\mathrm{pH}$-responsive microgels of controllable particle diameter by emulsion polymerization. Langmuir 22, 3381-3387 (2006).

28 Wishart, J. F. \& Neta, P. Spectrum and reactivity of the solvated electron in the ionic liquid methyltributylammonium bis(trifluoromethylsulfonyl)imide. J. Phys. Chem. B 107, 7261 (2003).

29 Kuwabata, S., Torimoto, T. \& Nakasawa, H. Availability of ionic liquid for electron microscopy. Kenbikyo 44, 61-64 (2009).

30 Torimoto, T., Okazaki, K., Kiyama, T., Hirahara, K., Tanaka, N. \& Kuwabata, S. Sputter deposition onto ionic liquid: simple and clean synthesis of highly dispersed ultrafine metal nanoparticles. Appl. Phys. Lett. 89, 243117 (2006).

31 Nakamoto, H. \& Watanabe, M. Brønsted acid-base ionic liquids for fuel cell electrolytes. Chem. Commun. 24, 2539-2541 (2007).

32 Debeljuh, N. J., Sutti, A., Barrow, C. J. \& Byrne, N. Phase transition of poly ( $N$-isopropylacrylamide) in aqueous protic ionic liquids: kosmotropic versus chaotropic anions and their interaction with water. J. Phys. Chem. B 117, 8430-8435 (2013).

33 Geisel, K., Isa, L. \& Richtering, W. Unraveling the 3D localization and deformation of responsive microgels at oil/water interfaces: a step forward in understanding soft emulsion stabilizers. Langmuir 28, 15770-155776 (2012).

34 Crassous, J. J., Rochette, C. N., Wittemann, A., Schrinner, M., Ballauff, M. \& Drechsler, M. Quantitative analysis of polymer colloids by cryo-transmission electron microscopy. Langmuir 25, 7862-7871 (2009)

35 Fujii, S., Armes, S. P., Araki, T. \& Ade, H. Direct imaging and spectroscopic characterization of stimulus-responsive microgels. J. Am. Chem. Soc. 127, 16808-16809 (2005).

36 Fujii, S., Dupin, D., Araki, T., Armes, S. P. \& Ade, H. First direct imaging of electrolyteinduced deswelling behavior of $\mathrm{pH}$-responsive microgels in aqueous media using scanning transmission x-ray microscopy. Langmuir 25, 2588-2592 (2009).

Supplementary Information accompanies the paper on Polymer Journal website (http://www.nature.com/pj) 